\title{
Communication difficulties following right hemisphere stroke: applying evidence to clinical management
}

\author{
Catherine Mackenzie \\ Department of Educational and Professional Studies, University of Strathclyde, \\ Glasgow
}

\section{Marian Brady}

Nursing, Midwifery and Allied Health Professions Research Unit

Glasgow Caledonian University, Glasgow

Correspondence to:

Professor C Mackenzie

Department of Educational and Professional Studies

University of Strathclyde

Southbrae Drive

Glasgow G13 1PP

01419503454

c.mackenzie@strath.ac.uk 


\begin{abstract}
Following reports in the 1960s that language may be affected by right hemisphere (RH) lesions, many limitations to effective communication in the right hemisphere damaged (RHD) population have been described and evidenced.
\end{abstract}

However, stereotypical portrayals and descriptions of carefully selected cases may be misleading as to the extent of communication deficits. In many of the parameters in which RHD patients are presented as typically impaired, e.g. discourse skills, a less severe picture may emerge where data from the non-brain damaged (NBD) population are considered, with age and education variables controlled. Subsequent to RHD, some people show deficit on some communication measures, but many of these communication behaviours are also present in some NBD adults. Thus diagnosis of deficit must be made with reference both to the healthy peer population and the individual's pre-lesion behaviour.

The authors' right RH stroke research programme includes studies of incidence of communication deficit, comparisons of RHD and NBD groups in various spoken discourse and comprehension tasks, comparison of RHD groups of different ages, detailed analysis of topic within discourse in RHD and NBD groups, family members' views of communication behaviour following RHD, and the natural course of communication change during the first year after RH stroke. The findings from several studies are summarised and used as the basis for management recommendations, which may guide future outcome research. There is an urgent need for the evaluation of communication management programmes, to determine whether therapists may with confidence offer an effective intervention service to those people whose communication skills are affected by RHD. 
Speech and language therapy clinicians may receive referrals of people who present with language and communication difficulties subsequent to lesions in either the left hemisphere ( $\mathrm{LH})$ or the right hemisphere (RH). Knowledge of the features of aphasia, associated mainly with LH damage, its diagnosis, incidence and prevalence, is considerably further advanced than is the case for the communication difficulties which may occur with RH damage (RHD) (Tompkins, Fassbinder, Lehman-Blake \& Baumgaertner, 2002). Recognition of how communication skills may be affected in RHD has a relatively short history, compared to that of aphasia. Eisenson (1962) and Critchley (1962) are credited with the identification that contrary to the prevailing view at the time, patients with RHD were not necessarily free of language impairment, though difficulties were less obvious than those in aphasia. Such observations were strengthened by evidence from split-brain studies that the disconnected RH could carry out basic language activities (Gazzaniga \& Sperry, 1967). Recognition of the RH's contribution to effective communication was later supported by modern neuroimaging (Knecht et al., 2000; Springer et al., 1999). The idea of a co-operative relationship between the two hemispheres, the LH viewed as analytic, and the RH responsible for a more holistic level of processing, was forwarded by Gardner (1977) and Nebes (1978). This dichotomy is now seen as an over-simplification, but the notion of effective communication requiring both hemispheres is firmly established.

As yet there is no generally accepted diagnostic label for the subtle and variable communication difficulties associated with RHD, though dyshyponoia (Paradis, 1998), pragmatic aphasia (Joanette \& Anslado, 1999) and apragmatism (Myers, 2001) have been suggested. Furthermore, though there is a long history of published evaluation of the outcome of interventions for aphasia (Kelly, Brady, Enderby (in progress), assessment of benefits of management for RH communication difficulties is scant, and evidence is confined to the level of expert opinion. Management recommendations for any population should be clearly linked to deficits which are firmly established in that population. A number of methodological issues affect the diagnostic process for the RHD population and these are discussed in this paper. 


\section{Communication deficit following right hemisphere damage}

\section{Approaches to identification of RH communication deficit}

Some anecdotal or summary accounts of the communication profile of individuals with RHD (e.g. Patterson \& Chapey, 2008; Zanini, Vorano \& De Luca, 1998) may have provided an impression that communication difficulties in this population are both more severe and more common than is the reality. Myers (1999) portrays the patient whom clinicians would regard as 'typical' as either an abrupt, disinterested and insensitive communication partner, who may take little account of social communication conventions, perhaps interrupting and failing to make eye contact, or alternatively as a verbose, rambling communicator whose discourse shows tangential associations. Ability to integrate and interpret incoming information may also be impaired, leading to difficulties with some aspects of comprehension. Prosodic, affective and cognitive impairments, including denial, attention deficit and neglect, may accompany and contribute to the communication disorder. Various investigations have testified to the presence of these communication features in some people with RH damage, but are characteristics such as these, often regarded as 'typical' in fact widespread in the RHD population?

As with other brain pathology populations, those with RHD are a heterogeneous group as regards lesion location and extent, and presence and severity of various communication difficulties, in addition to the many other factors which influence communication such as age, cognitive ability, educational level, time after lesion, individual response, and possibly handedness. Given the absence of an 'independent, theoretically and psychometrically sound measure for documenting the presence, nature, or severity of RHD language disorders' (Tompkins et al., 2002, p. 431), objectively identifying an appropriate population to study is problematical. If research participants are selected because of the clear presence of communication difficulties, or referral to speech and language therapy, these may well have come to professional attention because they are in fact group 'outliers' or even before brain damage may have had an extraordinary communication style (Brady, Armstrong \& Mackenzie, 2005). A second, alternative approach to the study of communication in RHD participants is to compare unselected cases, included on the basis of established 
neurological pathology, such as stroke, and their NBD peers, thus eliminating the risk of selection bias. Such a RHD group will not be confined to those whose communication is affected. Mean score comparisons thus inform on whether statistically significant differences are present at a group level, in addition to the range and distribution of scores in both populations. A third method, which avoids reducing the description of varied individuals to a group mean, is to gather the views of familiar communication partners as to whether communication competence has altered following brain damage, relative to premorbid status. All three approaches inform, but each has disadvantages.

\section{RHD investigation programme}

Findings from the authors' programme of investigation into the presence and nature of communication difficulty following RH stroke provide the foundations for the practice recommendations forwarded in this paper. The results of investigations of RHD performance in various communication tasks, including discourse, evaluated with reference to NBD peer populations, are reported. Also described are the findings from studies of the incidence of communication deficits in a large $\mathrm{RH}$ stroke group, communication presentation in older, relative to younger, RH stroke groups, family members' views of communication status following stroke, and the natural course of recovery. While the emphasis is the authors' research programme, reference is made to others' work where appropriate.

RHD participants for the studies referred to were drawn from sequential admissions to a large city hospital acute stroke unit with a catchment population of 225,000. Referred patients were admitted for a period of approximately 72 hours for inter disciplinary assessment, investigation and acute treatment, and are then discharged or transferred for rehabilitation and continuing care. First ever stroke confined to the RH was established through clinical presentation and computerised tomography or magnetic resonance imaging. Stroke was categorised as ischaemic or haemorrhagic and as total anterior circulation syndrome - TACI (cortical + subcortical lesion), partial anterior circulation syndrome - PACI (a) (cortical lesion anterior to Sylvian fissure), partial anterior circulation syndrome - PACI (p) (cortical lesion posterior to Sylvian fissure), lacunar syndrome - LACI (subcortical lesion) and posterior 
circulation syndrome - POCI (vertebrobasilar territory lesion) (Bamford, Sandercot, Dennis, Dunn \& Warlow, 1991).

Participant inclusion criteria included no history of other neurological or psychiatric problems, nor of drug or alcohol abuse, and hearing and vision judged adequate for assessment. Additional inclusion criteria were English as first language, performance within normal limits in a dementia screening assessment (Anomolous Sentences Repetition Test (ASRT), Weeks, 1988) and the Sentence Comprehension Test (Brookshire and Nicholas, 1993). While non-right handed participants have also been studied (Mackenzie \& Brady, 2004), all participants referred to in this paper were right handed. The timing of assessment is controlled. For some of the research, initial communication assessment was carried out at the acute, in-patient stage. Follow up assessments on patients who continued to fulfil the inclusion criteria (e.g. no further neurological event and ASRT scores continuing to be normal), were conducted at one, three, six and 12 months. Participants whose status did not permit acute assessment were first accessed at one month after stroke. For some of the research described, assessed points were one and six months only. Only three assessors have been involved throughout, all qualified speech and language therapists (SLTs): the stroke unit's SLT, as a member of the research team, and the authors. In some instances participants' close relatives contributed to the communication evaluation. No participants received communication treatment at any time during the period of study. NBD control participants (age, education and gender) were sourced via community, education, church groups, and personal contacts, and fulfilled all inclusion criteria, except that of the stroke event.

\section{Establishing incidence}

It has been estimated that around $50 \%$ of those with RHD show communication difficulties (Benton \& Bryan, 1996; Joanette \& Goulet, 1994), thus suggesting a higher prevalence than for aphasia, which is present in $33 \%$ of acute stroke patients (Laska, Hellblom, Murray, Kahan \& Von Arbin, 2001). Establishing incidence of communication difficulty will depend on many factors, including the professional background of the assessor, the aspect of communication studied, the time after brain damage, the sensitivity of the measures, and the appropriateness of normative data. A further important variable, not always controlled in RHD research, is the cause of 
brain damage, and combining participants with stroke and traumatic brain injury is likely to confuse the evidence (Paradis, 1998).

Mackenzie, Begg, Lees and Brady (1997) evaluated all consenting patients with confirmed diagnosis of first ever RH stroke, admitted to an acute stroke unit over a period of 14 months, who fulfilled the inclusion criteria detailed above. Spoken discourse was assessed in conversation and picture description (cookie theft: Goodglass \& Kaplan, 1983). Ratings were made of conversational verbal and nonverbal parameters (Burns, Halper \&Mogil, 1985). Counts of words and content units (literal and interpretive) and ratios of efficiency (word count relative to content unit count) and extraneous information (number of occurrences relative to word count) were calculated in picture description. Comprehension assessment included understanding of discourse (Brookshire \& Nicholas, 1993), inferential material (Bryan, 1994), metaphor (Bryan, 1994), and synonyms (Kay, Lesser \& Coltheart, 1992). At 3 months after stroke, data from 70 participants in the age range 36-88 years were available. Performance was appraised in relation to that of 189 NBD adults, aged 40-88, for whom test-retest, inter-rater and intra-rater reliability were established (Mackenzie, 2000a; 2000b). RHD and NBD participants were considered within three age groups (36-59, 60-74 and 75-88) and three educational levels (school leaving at minimum age, school leaving at certificate level, university or equivalent higher education). Where effects of age, education or gender were established in the normative data, the individual stroke participant was assessed in relation to scores for the appropriate grouping. Scores below the $10 \%$ ile NBD performance were taken as indicative of deficit. Figure 1 shows the proportion of participants with so defined low scores on the various measures.

\section{Figure 1 about here}

Conversational non-verbal communication (facial expression, eye contact, and intonation) was the most affected measure, with low scores in almost three quarters of participants at three months. The conversational verbal communication measure (initiation, verbosity, turn taking, referencing, topic maintenance) was impaired in $31 \%$ of those under 75 years, but only in $10 \%$ of those over 75 years. Thirty-five per 
cent had low interpretative unit scores in picture description and $30 \%$ had reduced or lengthy descriptions, the former being more common. The incidence of comprehension deficit at three months was between $21 \%$ and $44 \%$ on the various measures.

Twenty per cent of the RHD group showed a strong communication profile, performing not lower than the NBD 10\%ile in nine of the 10 measures. These participants did not differ in stroke location (cortical or subcortical), age or education, from those whose communication skills showed more obvious impairment $\chi^{2}$-age: 0.471, education: 0.014 , stroke: 2.506 , all n.s). Twenty-one per cent showed a generalised communication impairment, affecting conversational discourse, picture description and comprehension, with deficit scores in at least five of the 10 measures. Associations between this low performance and both younger age and education above minimum level were indicated $\left(\chi^{2}-\right.$ age: $3.921, \mathrm{p}=.048$, education $6.295, \mathrm{p}=$ $.012)$, but not in respect of stroke location $\left(\chi^{2}: 0.122\right.$, n.s. $)$.

\section{Influence of stroke classification}

Stroke was haemorrhagic in seven per cent of cases. There were no indications that communication profile was affected by aetiology being haemorrhagic or ischeamic, so data were combined. Unlike in aphasia, in which there are established associations between sites of left brain damage and distinctive communication profiles, differentiations according to stroke class or location are unusual in RHD studies..

According to Brookshire (2007), RHD group studies may comprise disproportionate numbers of those with anterior injuries as they may remain in hospital longer and so be available for recruitment to communication studies. This bias is avoided where participants are sourced in the first few days after stroke. The anterior damage categories, (PACI (a) and TACI), together accounted for $50 \%$ of the sample. Although volume of infarct is large in TACI, compared to other stroke classes, Mackenzie et al. (1997) did not find this subgroup, which comprised 34\% of the sample, more likely to show impaired performance than those with more restricted lesions. Within this subgroup, and within each of the major groupings, were individuals with strong and weak communication profiles. Joanette, Lecours, Lepage 
and Lamoureux (1983) suggested that more intense communication difficulty would be present in cortical than in subcortical damage. Mackenzie et al. (1997) found cortical lesions $(61 \%)$ were associated with lower scores in the picture description interpretive unit measure than was the case for subcortical lesions (Man-Whitney test $\mathrm{U}=137.5, \mathrm{p}=.01)$. Otherwise no relationships with stroke location were established. This finding may reflect the impact of subcortical damage on cortical pathways (Nadeau \& Gonzalez Rothi, 2001) or be supportive of Cappa, Papagno and Vallar's (1990) hypothesis of a right hemisphere subcortical role in language processing or in the cognitive processes which support communication. Bamford et al. (1991) noted that despite anatomically small lesions LACI patients may have persisting handicaps.

\section{The cognitive dimension}

Both stroke and traumatic brain injury, the main causes of acquired language and communication difficulty, may affect cognitive skills, such as attention, perception and memory, and low RHD cognitive test performance has been reported (Cherney \& Halper, 2002). Cognitive skills underpin language and communication. For example, memory impairment will influence discourse comprehension and ability to keep track of and participate in conversation. The language and communication difficulties of RHD patients tend to be evident with more complex and so cognitively demanding tasks, rather than the difficulties with basic language activities seen in aphasia. A common conclusion is that communication in RHD is less effective because of cognitive deficits (Cherney \& Halper, 1996).

Assessing cognitive skills of brain damaged people is notoriously difficult. Measures may be rendered unreliable because of neurological sequelae, especially where language is the medium of test administration and response (Keil \& Kaszniak, 2006), or may lack ecological validity (Chaytor \& Scmitter-Edgecombe, 2003). In the RHD research reported here, cognitive assessment was restricted to visual neglect, assessed by the Simple Test of Visual Neglect (Albert, 1973) and ASRT, a dementia screening assessment regarded by its author as a suitable tool 'to provide evidence or otherwise of cognitive deterioration' (Weeks, 1988, p.17). Participants were not excluded because of the presence of visual neglect, but care was taken in positioning visual assessment materials where a degree of neglect was present. ASRT score did not 
differ in the subgroups of those with strong and weak communication profiles $(U=$ 65.50 , n.s.).

RHD studies typically acknowledge the relevance of the cognitive processes and the potential for communication to be affected by cognitive involvement. However, with the exception of some associations with visuospatial neglect (Blake, Duffy, Myers \& Tompkins, 2002), and between working memory and a highly demanding discourse comprehension task (Tompkins, Bloise, Timko \& Baumgertner, 1994), correlations between cognitive and communication performance have rarely been established. Furthermore, a growing body of research suggests that specific links between RH damage and cognitive loss may be less strong than commonly believed. Brookshire (2007) points out that because a LH damaged control group is rarely included in RHD studies, it is not possible to differentiate the general effects of brain injury from hemisphere specific effects. Gillespie, Bowen and Foster's (2006) meta-analysis of memory impairment in RH stroke found performance to be superior to that of LHD for verbal recall and recognition tasks. McDonald (2000) found a RHD group to be significantly poorer than a NBD group on visuospatial tasks and a prose recall task, but otherwise the groups were not discriminated on tests of attention, executive function and memory, though some individuals with RHD did perform poorly. Rather than seeking links with specific cognitive contributions, Monetta and Joanette (2003) forward the view that the $\mathrm{RH}$ provides nonspecific support to the pool of cognitive resources or their allocation. Carrying out the most challenging communication tasks would thus be affected by a quantitative reduction in cognitive resources.

\section{Communication in elderly people with RHD}

RHD communication research has rarely considered the influence of age, despite much research which demonstrates its relevance across many tasks, including those designed for assessment of people with RHD (Zanini, Bryan, De Luca \& Bava, 2005). Mackenzie, Begg, Lees and Brady (1999) found that at one month after stroke, statistically significant differences between NBD $(n=40)$ and RHD $(n=36)$ groups of age below 75 years were present in many verbal discourse and comprehension measures. Furthermore, there were many similarities between the performance of this RHD group and very elderly NBD participants (age 75-88, $n=12$ ), consistent with 
observations regarding parallels between communication in RHD and healthy elderly people (Tompkins, 1995).

The majority of differences observed between NBD and RHD younger populations were not present in groups of age 75-88 $(\operatorname{NBD} n=20$, RHD $n=12)$. In these very elderly groups, the only measures where RHD scores were lower were conversational non-verbal communication and picture description interpretive unit counts (Man Whitney tests, non-verbal: $U=20.00, p<0.0001$, interpretive: $U=62.50, p=0.01)$. It is hypothesised that because of the cognitive deterioration associated with the normal aging process, effects of RHD are less marked in aged people than is the case with younger participants. This finding has important implications for intervention, as well as for diagnosis. Communication performance in the healthy adult population is different in those of advanced age, compared to middle aged and young elderly people. Where brain damage has occurred, some communication features which are in fact standard within the NBD population may be mistakenly thought to be a neurological consequence. Without age related normative data, communication intervention targets may be set for very elderly RHD patients which are no more appropriate than for the NBD population. Similarities between NBD and RHD elderly groups is likewise indicated by work of Blake (2006). Experienced speech and language therapists, who were familiar with RH disorders, showed poor diagnostic accuracy when blindly attributing discourse samples to either a healthy older adult or RHD group.

\section{Topic in discourse}

Given its ecological validity, strong arguments can be forwarded for the evaluation of discourse in any communication disordered population. Descriptions of the discourse of people with RHD include difficulties with topic use and management. Using severity rating scales to assess conversational verbal skills, Mackenzie et al. (1997) found an interaction between topic maintenance scores and stroke status in those under age 75. Stroke participants were less likely to maintain topic than their NBD peers. The accruing of firm evidence with regard to topic skills in RHD has been limited by the use of such qualitative rating scales, or by analyses of single cases or small groups, with inadequate control data from the NBD peer population. 
Topic management and use by 17 patients with RH stroke, drawn from consecutive hospital admissions, and 51 gender, age and education matched NBD participants was explored in semi-structured conversation (Brady, Mackenzie \& Armstrong, 2003) and in procedural and descriptive discourse (Brady et al., 2005). No widespread or consistent differences between RHD and NBD groups were shown using quantitative, objective analytical measures. Significant differences were present in some discourse features, including repetition and fillers (e.g. 'eh', 'em', 'you know') at one month after stroke. The RHD group had fewer of these component ( $t$ tests, $p$ values ranging from 0.001 to 0.047 ), which skilled communicators have been observed to employ to manipulate discourse (Schegloff, 1987). By six months after stroke, the RHD group did not differ from the NBD group in their use of repetition, but some differences remained in relation to their use of fillers ( $\mathrm{p}$ values ranging from 0.0001 to 0.045 ). The RHD group were not observed to have any difficulty staying on topic. There was also a suggestion, which requires confirmation through more targeted investigation, that compared with the NBD group, the RHD group used less structure within topics (subtopic structures) a month after stroke, in some samples ( $\mathrm{p}=0.038 ; 0.028 ; 0.038)$.

Despite frequent descriptions in the literature of rambling and disorganised speech following RHD, there was little sign of consistent patterns of difference between the individuals' with RHD and the matched NBD individuals' use of topics during discourse. Some individuals with RHD had interesting topic patterns, but this was also the case for some NBD participants. For example, when asked to 'Tell me about your family' significantly more NBD individuals moved from the topic of 'family' to, for example, discuss their career or their interest in swimming. In contrast the RHD group did not deviate at all from the topic of 'family'. During the procedural discourse sample of 'how to make a sandwich' the opposite was true. None of the NBD participants deviated from the discourse task while significantly more of the RHD group did. However these differences were not present across all prodedural discourse tasks. McDonald (2000) reported RHD and NBD groups to be similar on number and relevance of steps in procedural discourse, with no evidence of increased tangentiality in the RHD group. For the individual with RHD whose topic behaviour appears remarkable, it is therefore important to seek to establish whether or not this characteristic was present before stroke. As with many investigations of 
communication and cognition in RHD, recent evidence thus indicates that features which have often been regarded as discriminative are by no means typical of the RHD population.

Further additional indication that the prevalence of some communication difficulties in RHD may have been overstated comes from Blake et al. (2002). In a review of the hospital records of 123 RHD patients, in only $16 \%$ of cases, professionals from neurology, neuropsychology, speech-language pathology or occupational therapy had noted deficits in interpersonal interaction (incorporating inappropriate pragmatics). Comparable is Odell, Wollack and Flynn's (2005) audit of the progress of 101 RHD patients who received speech and language therapy. Median performance on a social interaction measure at initial assessment was near to ceiling and at ceiling on final assessment, though some individuals clearly had pronounced impairment.

\section{The family view}

Since many of the communication features often considered indicative of RHD are present in some NBD people also, evaluation should take account of the individual's pre-stroke status. Someone who is a familiar communication partner both currently and before stroke, will have a view as to whether and how communication has changed. Mackenzie, Brady, Begg and Lees (2001) adopted this approach with 23 RHD participants, who had communication deficit across a range of clinical tasks one month after stroke. Ratings of change relative to pre-stroke ability were made by relatives on a 100 point scale using the 16 items of the Communicative Effectiveness Index (Lomas, Pickard, Bester, Elbard, Finlayson, \& Zoghaib, 1989) plus five additional items, included for their relevance to the communication difficulties in the RHD population. At this one month point, seven of the evaluated communication activities were regarded as impaired by over one third of the raters. Of these seven, six are relevant to conversational skills: 'being part of a conversation where it is fast', 'describing or discussing something in depth' 'getting involved in group conversations', communicating emotions', 'making clear who/what he/she is talking about' and 'getting and sticking to the point in conversation' The extent of perceived loss across the group of raters was not high, with 'being part of a conversation where it is fast' attracting the highest mean loss (24\%). Despite having low scores on a 
number of communication tasks, six participants were rated by their relatives as unchanged. Such data, deriving from communication in everyday situations, rather than in clinical testing, confirms the presence of communication difficulty in the RHD population, but indicates that even where low scores are present in clinical tasks, some familiar communication partners identify no change from the pre-stroke situation.

\section{Change over time}

The RHD populations described in this paper received no intervention for communication difficulties, and are thus suitable populations in which to evaluate the natural course of recovery.

The progress of 17 patients, who were impaired both in terms of clinical communication assessment scores and relatives' impressions, was examined at one, three, six and 12 months after stroke (Mackenzie et al., 2001). Clinical task performance was evaluated as a composite deficit score reflecting performance over a range of measures, and not in relation to individual task scores. This approach was adopted for validity of comparison with relatives' ratings which were made over various situations. Significant improvements were present between one and six months and between one and 12 months, with a strong trend in the direction of improvement between six and 12 months (Wilcoxon tests: 1 and 12 months $Z=-2.97$, $\mathrm{p}=.003,1$ and 6 months $\mathrm{Z}=-2.20, \mathrm{p}=.028,6$ and 12 months $\mathrm{Z}=-1.90, \mathrm{p}=.057)$. It is not within the scope of this paper to postulate on the many variables which may contribute to improved communication performance, but these results are consistent with current views of the brain as a dynamic structure which is constantly changing in a way that may enhance cognitive skills (Mlcoch and Metter, 2008).

For the family ratings (Mackenzie et al., 2001), significant improvements were present between one and three months, and between one and six months, with a trend towards improvement between three and six months (Wilcoxon tests: 1 and 3 months $Z=-1.99, p=0.47,1$ and 6 months $Z=-2.53, p=.011,3$ and 6 months $Z=-1.81, p=$ .070). A reverse of the direction of change was present between six and 12 months, when significant increase in communication difficulty was perceived $(Z=-2.67, p=$ .008). One and 12 month comparison showed equivalence of rated loss in relation to 
prestroke competence $(\mathrm{Z}=0.781, \mathrm{p}=.435)$. It may be that family become more aware of communication difficult within the second period of six months, as additional situations present themselves when individuals return to their homes, work and social circles, This, combined with the reality of anticipated full recovery not having taken place, may lead to a reduction in perceived competence.

Responses during three conversational, three procedural and a picture description task were examined in eight RHD individuals, aged 62-79, at one and six months after stroke (Brady, Armstrong \& Mackenzie, 2006). Detailed analyses included length, syntactic complexity, physical and illustrative gestures, verbal disruption, cohesion and topic coherence. Whereas the participant group in the Mackenzie et al. (2001) study comprised RHD patients with demonstrated communication deficit, this group were unselected in that respect. Despite high levels of intra-rater reliability in the analyses, no widespread significant differences were evident over time in the language and discourse features measured, and there were no apparent task effects. Group size was small in this study, and communication may not have been affected by stroke in at least some participants. Also communication parameters were examined individually, as distinct from summing deficit scores across a range of tasks, as in Mackenzie et al. (2001), so subtle changes may not have been discerned.

The research outlined in this paper demonstrates that stroke to the right hemisphere undoubtedly leaves some people less competent in communication than their peers, or relative to their pre-stroke status, but communication difficulty following RHD is not universal, nor necessarily severe. Many of the communication features which Myers (1999) reported that clinicians would regard as 'typical' following RHD (see above), were present in some individuals, but by no means will clinicians see these in all the RH stroke population. Furthermore the difference from pre-stroke communication behaviour may be slight. For some behaviours, and especially in aged people, RHD and NBD groups cannot be distinguished by their communication. When considering intervention, the clinician should note the range of performance present in the individual's age and educational grouping and also consider that what appear to be deficits may have been characteristic of the individual's communication before stroke. This may be especially important for very elderly people. Those for whom intervention is relevant are more likely to be under, than over, age 75 . 
Although the communication difficulties in RHD tend to be less obvious than those of aphasic people, they may nevertheless cause both disability, particularly for those whose employment or leisure pursuits require effective communication skills, and also distress to the individual and his/her family.

\section{Management implications}

Involvement of relatives or other caregivers is an essential component of the management of communication difficulties. They may provide information which contributes to diagnosis, and may be offered education and advice which facilitates their understanding of communication behaviours and consequently their ability to support and maximise communication exchanges. Relatives or caregivers may also be active participants in the intervention programme, following on from goal setting in which they, the patient, and other relevant professionals, such as occupational therapists, collaborate.

Mackenzie et al. (2001) found that for RHD patients with low clinical task performance, the presence of communication difficulty in everyday situations is confirmed by some, but not all relatives. Prestroke performance for these participants may have been low, in which case intervention is not appropriate. It is also possible that some relatives are insensitive to changes in communication, or have unconsciously altered their own communication in a facilitative way, or now make fewer language and communication demands. Van Lancker (1997) suggests that because the fundamental language processes of phonology, semantics and syntax are likely to be relatively intact, family may interpret communication changes as uncooperativeness or deliberately difficult behaviour. Tompkins, Lehman, Wyatt and Schulz (1998) refer to the stigmatisation which may be present, with RHD patients perhaps regarded by family and friends as inappropriate, uncaring or bizarre. According to Paradis (1998) the communication difficulties associated with RHD may constitute 'a social handicap at least as significant as aphasia.' (Paradis, 1998, p.7). The lack of awareness of deficit, or of the reactions of others, which may be associated with RHD, may further frustrate communication partners. Such 
observations underline the need for involvement of family members throughout the assessment, diagnosis and management process.

Tompkins (1995) referred to increasing numbers of adults with RHD in speech and language therapy (SLT) caseloads in the USA. Blake et al (2002) reported $45 \%$ of a hospital chart review dataset to have been referred to SLT. No information is provided as to reason for referral, which may have included dysarthria and dysphagia. In the UK, referral of RHD patients to SLT because of communication difficulty, rather than motor speech or swallowing disorder, is still relatively unusual. Van Lancker (1997) described rehabilitation of the communicative deficits arising from RHD as a 'much needed endeavour' (P5) and referred to the existence of numerous relevant treatment programs. Odell, Wollack and Flynn (2005) reviewed functional outcome in over 100 RHD patients and found a relationship between amounts of communication treatment and communication score gain, with greater improvement associated with more numerous sessions. Some treatments for visual neglect which incorporate scanning during language tasks (Blake, 2005) and some case studies on use of emotional prosody (Leon, et al., 2005; Rosenbek, et al., 2004) have shown task gains. Otherwise there is very little reliable evidence of language or communication treatment benefit for the RHD population.

\section{Approaches to intervention}

Two approaches to treatment, used singly or in combination, have been advocated for RHD communication difficulties. In the process-specific approach, the cognitive processes, such as attention and memory, which are thought to be impaired, and influencing communication, are targeted. The functional approach directly targets communication, incorporating strategies which may compensate for cognitive process impairments. Ylvisaker, Hanks and Johnson-Greene (2002) argue that there is no substantive evidence that decontextualised cognitive process exercises lead to improvement of the process and performance in real world tasks. In the management of traumatic brain injury they promote intervention which is contextualised from the outset, thus emphasising relevant social activities. Given the paucity of established correlation between cognitive and communication performance in RHD, we contend that where the aim is to improve communication, therapy should centre on 
communication. If during communication there is a difficulty in sustaining attention, or remembering, or planning, or problem solving, then working towards improvement of this should be tackled in realistic communication activities, especially interactive discourse. For example, where attention deficit hinders verbal comprehension or expression, communication treatment activities may be systematically manipulated in length, and be developed to incorporate the distractions and competing demands which are typical of everyday situations.

\section{Targeting non-verbal communication}

Impairments in aspects of non-verbal communication, relative to NBD peers, appear to be prevalent and persisting in RHD, affecting $65 \%$ of participants at one year after RH stroke (Mackenzie et al., 1997). Significant difficulties in this area of communication are present in all age groups (Mackenzie et al., 1999). In conversation the 'typical' RHD patient has limited eye contact, restricted facial expression and monotony of intonation. This may be linked to relatives rating communication of emotions as impaired (Mackenzie et al., 2001). The starting point for intervention is identification and awareness of the importance of facial expression, eye contact and intonation, and what is signalled through these behaviours, and by what means. We show interest in our communication partner and what we are communicating in many ways, including non-verbally. Surprise, criticism, anger and sadness are conveyed in part through intonation pattern and facial expression. Video examples and role plays can be used to illustrate where a speaker or listener communicates interest, disinterest and varied emotions, not through the words spoken, but through the manner of speaking, facial expression, gestures or eye contact pattern. Examples may be included where non-verbal messages are not consistent with what is being conveyed verbally. Therapist and patient discussion, with evaluation, of such impersonal examples, lays the foundation for appraisal and monitoring of the patient's non-verbal communication. This can be approached through video-recorded practice of appropriate facial expression, gestures, eye contact and intonation, in relation to differing situations and circumstances, followed by evaluation by therapist and patient. Success is then affirmed and revision attempted as appropriate. Compensatory strategies and cues may be introduced, such as an agreed reminding signal from the therapist or main communication partner, or a written cue card or illustration which the patient keeps in view. It should be recognised that there may be complex and 
varied explanations for non-verbal communication deficit, which extend beyond the realms of pragmatics. For some RHD patients, motor difficulties and emotional status may limit the appropriateness of these suggestions, or may indicate a requirement for a modification of methods.

\section{Targeting verbal conversational parameters}

A similar approach may be adopted where there are verbal conversational changes, such as unclear referencing, difficulties in management of topic, infringements of turn taking conventions, including turn length or failures to initiate conversation. As with non-verbal communication intervention, establishing awareness of the relevant parameter(s) and the identification of 'appropriate' and 'inappropriate' contributions is an initial treatment goal. Where several behaviours contribute to a communication style which is not effective, initially one or at most two should be targeted, with others introduced as appropriate. Written as well as spoken examples, whether recorded or actively role played, can be utilised. Where written material is used, liaison with occupational therapy may be required, so that account is taken of any visuo-perceptual difficulties which may be influencing performance. Compensatory orienting strategies, such as a verbal reminder or the inclusion of a left vertical coloured line, may be appropriate.

Examples from real life, such as those taken from television programmes, have more validity than workbook stimuli. Following awareness training, therapy proceeds to the practice stage, which may be approached by patient(s) and therapist taking turns at talk on a given topic. In view of the finding of Bloom, Borod, Obler and Gerstman (1993) that emotional content may negatively affect pragmatic performance in RHD, material which is emotionally neutral might precede that which is more emotionally challenging for the individual. The method of sequential contributions at talk may be used where conversational initiation or appropriate turn taking are targeted, but is also suitable for other goals. If reducing verbosity is an aim, a limit can be set on each contribution, such as one idea, or one sentence. As therapy progresses and length of response is less prescribed, the use of agreed cues, such as a hand signal, may assist the patient in recognising that a turn should be taken or relinquished. As contribution length is then increased it is important that there is a corresponding addition in 
information or ideas expressed. In this respect, Varley's (1997) report of a man with communication difficulties subsequent to a right heaemorrhagic lesion is salutary. Following treatment her patient showed improvement in monitoring his own discourse for irrelevancies, and also a reduction in verbosity in description tasks. However these did not appear to be entirely positive changes in that decrease of excess output drew attention to a paucity of ideas. The patient and his wife thought this style less acceptable than the previously lengthy discourse, which led Varley to consider a different orientation to treatment.

The sequential turn approach may be used where topic maintenance is the target, with the aim of both partners making a contribution which is relevant to the topic. If referential clarity is targeted, the topic for discussion can include a situation which involves a variety of people and places. Evaluation of the recorded practice should follow, with revision of turns where appropriate. The therapist's turns should include examples of both good and poor style. As with attention to non-verbal parameters, prompts such as cue cards may act as reminders. Conversational practice of this type, on a turn by turn basis, may be augmented by providing opportunities of other situations where appropriate contribution, clarity, relevance and efficiency of expression can be practised and monitored, such as waiting room contacts and telephone conversations. Semi structured, topic directed interview situations also provide useful practice, with gradual reduction of the amount of structure provided by the introduction stimulus (using increasingly broader discourse stimuli). Treatment might gradually progress towards stimuli that are representative of natural conversational interaction, where judgements of relevance and construction of contributions are more subjective, and made on the basis of the conversational partner. Perceived deviations from topic, clarity of message or appropriateness of length can be jointly reviewed and discussed. To guard against bias, formal evaluation of such outcomes should involve the use of blinded listener ratings. Rehabilitation of such behaviours is likely to be reliant on the degree to which the patient is aware of the deficits, and his/her ability to identify the points of departure from discourse topic, firstly on review and then in real-time. It may also be necessary to encourage the patient to incorporate fillers such as 'em' and 'eh' within the discourse, which will 
provide time for review of the discourse to date and planning of the following discourse.

\section{Targeting narrative discourse}

Based on the finding that around one third of RHD patients had low interpretative unit scores in picture description, or reduced or lengthy descriptions (Mackenzie et al., 1997), it may reasonably be concluded that for some patients an aim of therapy might be to increase the amount of relevant information in narrative discourse. The stimuli may be picture, auditorilly presented story, or video material watched by therapist and patient together, with the goal of interpreting and effectively describing the events and situations portrayed. Main ideas and details are listed and distinguished, and possible interpretations and inferences discussed. Events or ideas are thus established and ordered, using questioning and cueing to elicit additional material, an introduction and conclusion added, and with the aid of such script the patient then produces the narrative. A further stage is the elimination of the scripted cues. The response should be recorded for evaluation by patient and therapist, and revisions made as required. Throughout this process both therapist and patient have access to the stimulus to be described. Removing the therapist's view of the stimulus, or ensuring this is not familiar to the therapist, places additional requirements on the patient to communicate full information (sometimes referred to as constraint induced therapy), and renders requests for clarification and expansion more natural. A similar organisational and practice structure may be used at a later point for the relating of experiences which the therapist has not shared. Taking notes at the time of the event and/or at the stage of narrative planning may be a useful compensatory strategy to aid recall and response structuring.

Where the patient with RHD provides information which is incomplete or otherwise lacks clarity, consideration should be given to the possibility that comprehension is impaired. In this case additional attention may be given to understanding the stimulus material prior to the story retelling goals. Material may be included which incorporates for example the need to infer and to understand metaphor and comprehension, verified through yes/no or brief response questions. 


\section{Group therapy}

Intervention such as the above may be carried out on an individual basis, but more natural interactions are likely in group situations. In family ratings, involvement in group conversations was one of the communication situations thought to be most frequently affected by RH stroke (Mackenzie et al., 2001). Groups provide opportunities for discourse with a range of communication partners, and differing communication styles. Peer support may also be beneficial. Cherney and Halper (2007) promote a group approach for RHD patients as being suitable for all levels of care and severity. A variety of group types are outlined, including pragmatics and life skills, the latter run on an inter-disciplinary basis. As with individual therapy, Cherney and Halper (2007) emphasise the need for studies to determine the efficacy of group techniques with the RHD population.

\section{Evidence of benefit}

Evidence of benefit for communication intervention and related management is accumulating for some adult acquired neurological disorders (Royal College of Speech and Language Therapists, 2005). In RHD communication difficulties it would appear that the robust evidence clinicians require to guide and justify their practice does not exist, due to a puzzling absence of methodologically sound research. This may be due in some part to the difficulty of objectively distinguishing impairment from 'normality'. SIGN (Scottish Intercollogiate Guidelines Network) methodology (SIGN 2008), identifies eight levels of evidence to guide practice in the National Health Service, graded from $1^{* *}$ (high quality meta-analyses, systematic reviews of randomised controlled trials or randomised controlled trials with a very low risk of bias), to 4 , the lowest level (expert opinion). The current status of 'evidence' for intervention for RHD communication difficulties can be placed essentially at this lowest level, which allows for recommendations for practice to be made on the strength of experience, but without a body of supporting studies which demonstrate positive outcomes.

The recommended standards of clinical outcome testing specify well controlled single case or small single group studies, without external controls, as the first necessary stage in a five phase process of evaluating efficacy, effectiveness and efficiency 
(Robey and Schultz 1998). The randomised control trial may be the 'gold standard' methodology for establishing the value of intervention, but given the paucity of reliable evidence for treatment related improvement of RHD communication deficits, it is necessary to first define the components of the intervention and demonstrate that positive outcomes are achievable. To maximise the usefulness of phase one study findings, methodological rigour is essential. Participants with communication change subsequent to RH stroke should be identified with reference to appropriate age and education data and also with regard to the reports of familiar communication partners. The intervention approach should be thoroughly described, to permit replication with larger, well defined participant samples, at which stage the inclusion of control participant groups and procedures are justifiable. Even at the preliminary phases, evaluation of response to the intervention must be scrupulous, using valid and reliable outcome measures.

The ideas for management forwarded here arise from research evidence regarding the presence of some communication difficulties in some people with RH stroke. This is not intended as a complete consideration of either the communication difficulties which may be present, or of methods of treatment. The challenge remains to forward the results of therapy, using reliable, objective, and functionally relevant measures. 


\section{Acknowledgements}

The authors acknowledge with thanks the contributions of their research colleagues,

Thia Begg, Kennedy R Lees, and Linda Armstrong, and funding from the Stroke Association, University of Strathclyde and the Chief Scientist Office. The views expressed here are those of the authors and not necessarily those of the Chief Scientist Office. 


\section{References}

Albert, M. L. (1973). A simple test of visual neglect. Neurology 23, 658-664

Bamford, J., Sandercot, P., Dennis, M., Dunn, J. \& Warlow, C. (1991).

Classification and natural history of clinically identifiable subtypes of cerebral infarction. Lancet, 337, $1521-1526$.

Benton, E. \& Bryan, K. (1996). Right cerebral hemisphere damage: Incidence of language problems. International Journal of Rehabilitation Research, 19, 4754.

Blake, M. L.(2005). Right hemisphere syndrome. In L L LaPointe (Ed.) Aphasia and Related Neurogenic Language Disorders (pp. 213-224). New York: Thieme.

Blake, M. L. (2006). Clinical relevance of discourse characteristics after right hemisphere brain damage. American Journal of Speech-Language Pathology, $15,255-267$

Blake, M. L., Duffy, J. R., Myers, P.S. \& Tompkins, C. A. (2002). Prevalence and patterns of right hemisphere cognitive/communicative deficits: Retrospective data form an inpatient rehabilitation unit. Aphasiology, 16, 537-547.

Bloom, R., Borod, J., Obler, L. and Gerstman, L. (1993). Suppression and facilitation of pragmatic performance: Effects of emotional content on discourse following right and left brain damage. Journal of Speech and Hearing Research, 36, 1227-1235.

Brady, M., Armstrong, L. \& Mackenzie, C. (2005). Further evidence on topic use following right hemisphere brain damage: procedural and descriptive discourse. Aphasiology, 19, 731-747. 
Brady, M, Armstrong, L, Mackenzie, C. (2006). An examination over time of language and discourse production abilities following right hemisphere brain damage. Journal of Neurolinguistics. 19, 39-58.

Brady, M., Mackenzie, C. \& Armstrong, L. (2003). Topic use during right hemisphere brain damage during three semi-structured conversational discourse samples. Aphasiology, 17, 881-904.

Brookshire, R.H. (2007). Introduction to Neurogenic Communication Disorders, $7^{\text {th }}$ edition. St Louis: Mosby Elsevier.

Brookshire, R.H. and Nicholas, L.E. (1993). Discourse Comprehension Test. Tucson: Communication Skill Builders.

Bryan, K.L. (1994). The Right Hemisphere Language Battery. London: Whurr.

Burns, M.S., Halper, A.S. \& Mogil, S.I. (1985). Clinical Management of Right Hemisphere Dysfunction. Maryland: Aspen.

Cappa, S.F., Papagno, C. \& Vallar, G. (1990). Language and verbal memory after right hemisphere stroke: A clinical CT scan study. Neuropsychologia, 28, 503 -509 .

Chaytor, N. \& Scmitter-Edgecombe, M. (2003). The ecological validity of neuropsychological tests: A review of the literature on everyday cognitive skills. Neuropsychology Review, 13, 181- 197.

Cherney, L.R. \& Halper, A.S. (1996). A conceptual framework for the evaluation and treatment of communication problems associated with right hemisphere damage. In A.S. Halper, L.R. Cherney \& M.S. Burns (Eds.) Clinical Management of Right Hemisphere Dysfunction. (pp. 21-27). Maryland: Aspen. 
Cherney, L.R. \& Halper, A.S. (2002). Performance of subjects with right hemisphere stroke on the mini-mental state examination. Brain and Language, 83, 30-32.

Cherney, L.R. \& Halper, A.S. (2007). Group treatment for patients with right hemisphere damage. In R.J. Elman (Ed.). Group Treatment of Neurogenic Communication Disorders: The Expert Clinicians' Approach. (pp.269-296). San Diego: Plural Publishing.

Critchley, M. (1962). Speech and speech-loss in relation to duality of the brain. In V.B. Mountcastle (Ed.). Interhemispheric Relations and Cerebral Dominance. Baltimore. (pp. 208-213). Baltimore: John Hopkins Press.

Eisenson, J. (1962). Language and intellectual modifications associated with right cerebral damage. Language and Speech, 5, 49 - 53.

Gardner, H. (1977) The Shattered Mind: the Person After Brain Damage. London: Routledge and Kegan.

Gazzaniga, M.S. \& Sperry, R.W. (1967). Language after section of the cerebral commisures. Brain, 90, 131- 148.

Gillespie, D.C., Bowen, A. \& Foster, J.K..(2006). Memory impairment following right hemisphere stroke: A comparative meta-analytic and narrative review. The Clinical Neuropsychologist, 20, 59-75.

Goodglass, H. \& Kaplan, E. (1983). The Boston Diagnostic Aphasia Examination. Philadelphia: Lea and Febiger.

Joanette, Y. \& Anslado, A. I. (1999). Clinical note: acquired pragmatic impairments and aphasia. Brain and Language, 68, $529-534$.

Joanette, Y. \& Goulet, P. (1994). Right hemisphere and verbal communication: conceptual, methodological and clinical issues. In M.L.Lemme (Ed.). Clinical Aphasiology Vol 22 (pp.1-23). Austin: Pro-Ed. 
Joanette, Y., Lecours, A.R., Lepage, Y. \& Lamoureux, M. (1983). Language in righthanders with right-hemisphere lesions: A preliminary study including anatomical, genetic and social factors. Brain and Language, 20, $217-248$.

Kay, J., Lesser, R. \& Coltheart, M. (1992). Psycholinguistic Assessments of Language Processing in Aphasia (PALPA). London: Erlbaum.

Keil, K. \& Kaszniak, A. W. (2002). Examining executive function in individuals with brain injury: A review. Aphasiology, 16, 305-335.

Kelly, H, Brady, M, \& Enderby, P (in progress) Speech and language therapy for aphasia following stroke, Cochrane Systematic Review.

Knecht, S. Drager, B., Deppe, M., Bobe, L., Lohmann, H., Floel, A., et al. (2000). Handedness and hemispheric language dominance in healthy humans. Brain, $123,2512-2518$

Laska, A.C.,Hellblom, A., Murray, V., Kahan, T. \& Von Arbin, M. (2001). Aphasia in acute stroke and relation to outcome. Journal of Internal Medicine, 249, 413-422.

Leon, S. A., Rosenbek, J. C., Crucian, G. P., Hieber, B., Holiway, B., Rodriguez, A.D., et al. (2005). Active treatments for aprosodia secondary to right hemisphere stroke, Journal of Rehabilitation Research and Development, 42, 93-102.

Lomas, J., Pickard, L., Bester, S., Elbard, H., Finlayson, A. \& Zoghaib, C. (1989). The communicative effectiveness index: Development and psychometric evaluation of a functional communication measure for adult aphasia. Journal of Speech and Hearing Disorders, 54, 113-124.

Mackenzie, C. (2000a).Adult spoken discourse: the influences of age and education. International Journal of Language and Communication Disorders, 35, 269285. 
Mackenzie, C. (2000b). The relevance of education and age in the assessment of discourse comprehension. Clinical Linguistics and Phonetics, 14, 151-161.

Mackenzie, C., Begg, T., Lees, K. R. \& Brady, M. (1997). Right hemisphere stroke: Incidence, severity and recovery of language. Final Report to the Stroke Association

Mackenzie, C., Begg, T., Lees, K.R. \& Brady, M. (1999). The communication effects of right brain damage on the very old and the not so old. Journal of Neurolinguistics, 12, 79-93.

Mackenzie, C, Brady, M., Begg, T. \& Lees, K.R. (2001). Communication ability following right brain damage: the family perspective. Advances in Speech Language Pathology, 3, 81-96.

Mackenzie, C, \& Brady, M 2004 Communication ability in non - right handers following right hemisphere stroke. Journal of Neurolinguistics, 17, 301-313

McDonald, S. (2000). Exploring the cognitive basis of right-hemisphere pragmatic language disorders. Brain and Language, 75, 82-107.

Mlcoch, A. G. \& Metter, E. J. (2008). Medical aspects of stroke rehabilitation In R Chapey (Ed.) Language Intervention Strategies in Aphasia and Related Neurogenic Communication Disorders. (pp.42 - 63). Philadelphia: Lipincott Williams and Wilkins.

Monetta, L. \& Joanette, Y. (2003). Specificity of the right hemisphere's contribution to verbal communication: The cognitive resources hypothesis. Journal of Medical Speech-Language Pathology, 11, 203-211.

Myers, P. S. (1999) Right Hemisphere Damage: Disorders of Communication and Cognition. London: Singular.

Myers, P. M. (2001) Towards a definition of RHD syndrome. Aphasiology, 15, 913 - 
918. 
Nadeau, S.E. \& Gonzalez Rothi, L.J. (2001). Rehabilitation of subcortical aphasia. In R Chapey (Ed.) Language Intervention Strategies in Aphasia and Related Neurogenic Communication Disorders. (pp.457-471). Philadelphia: Lipincott Williams and Wilkins.

Nebes, R.D. (1978). Direct examination of cognitive functions in the right and left hemispheres. In M. Kinsbourne (Ed.) Asymmetrical Function of the Brain. (pp.99-137). London: Cambridge University Press

Odell, K.H., Wollack, J. A. \& Flynn, M. (2005). Functional outcomes in patients with right hemisphere brain damage. Aphasiology, 19, 807-830

Paradis, M. (1998). The other side of language: Pragmatic competence. Journal of Neurolinguistics, 11, 1-10.

Patterson. J. P. \& Chapey, R. (2008). Assessment of language disorders in adults. In R. Chapey (Ed.), Language Intervention Strategies in Aphasia and Related Neurogenic Communication Disorders, $5^{\text {th }}$ edition. (pp. 64-160). Philadelphia: Lippincott, Williams \& Wilkins.

Robey, R. R. and Schulz, M. C. (1998). A model for conducting clinical-outcome research: an adaptation of the standard protocol for use in aphasiology. Aphasiology, 12, 787-810.

Rosennbek, J.C., Crucian, G.P., Leon, S.A., Hieber, B., Rodriguez, A.D., Holiway, B., et al. (2004). Novel treatments for expressive aprosodia : A phase I investigation of cognitive linguistic and imitative interventions. Journal of the International Neuropsychological Society, 10, 786-793.

Royal College of Speech and Language Therapists (2005). Royal College of Speech and Language Therapists Clinical Guidelines Bicester: Speechmark Publishing 
Scottish Intercollogiate Guidelines Network (2008). SIGN 50. A Guideline Developer's Handbook. Edinburgh: SIGN

Schegloff, E.A. (1987). Recycled turn beginnings: a precise repair mechanism in conversation's turn-taking organisation. In G. Button and J.R.E. Lee (Eds.) Talk and Social Organisation. (pp. 70 - 85). Clevedon: Multilingual Matters.

Springer, J. A., Binder, J. R., Hammeke, T. A., Swanson, S. J., Frost, J. A., Bellgowan, P. S., et al. (1999). Language dominance in neurologically normal and epilepsy subjects: a functional MRI study. Brain, 122, 2033-2046.

Tompkins, C.A. (1995). Right Hemisphere Communication Disorders: Theory and Management. San Diego: Singular.

Tompkins, C.A., Bloise, C.G.R., Timko, M.L.\& Baumgaertner, A. (1994). Working memory and inference revision in brain-damaged and normally aging adults. Journal of Speech and Hearing Research, 37, 896-912.

Tompkins, C.A, Fassbinder, W., Lehman-Blake, M.T. \& Baumgaertner, A. (2002). The nature and implications of right hemisphere language disorders: Issues in search of answers. In A E Hillis (Eds.) The Handbook of Adult Language Disorders. (pp. 429-448). New York: Psychology Press.

Tompkins, C.A., Lehman, M.T., Wyatt, A.D. \& Schulz, R. (1998). Functional outcome assessment of adults with right hemisphere brain damage. Seminars in Speech and Language, 19, 303-321.

Van Lancker, D. (1997). Rags to riches: Our increasing appreciation of cognitive and communicative abilities of the human right cerebral hemisphere. Brain and Language, 57, 1 -11. 
Varley, R. (1997). The treatment of communication deficits following right hemisphere lesion. In S. Chiat, J. Law \& J. Marshall (Eds.) Language Disorders in Children and Adults: Psycholinguistic Approaches to Therapy. (pp. 218-234). London: Whurr.

Ylvisaker, M., Hanks, R. \& Johnson-Greene, D. (2002). Perspectives on rehabilitation of individuals with cognitive impairment after brain injury: Rationale for reconsideration of theoretical paradigms. Journal of Head Trauma Rehabilitation, 17, 191-209.

Weeks, D.J. (1988). The Anomolous Sentences Repetition Test Windsor: NFER Nelson.

Zanini, S., Vorano, L. \& De Luca, G. (1998) Pragmatic disorders of verbal communication. Italian Journal of Neurological Sciences, 19, S27-S28

Zanini, S., Bryan, K., De Luca, G. \& Bava, A. (2005). The effects of age and education on pragmatic features of verbal communication: Evidence from the Italian version of the Right Hemisphere Language Battery (I-RHLB). Aphasiology, 19, 1107-1133. 
Figure 1

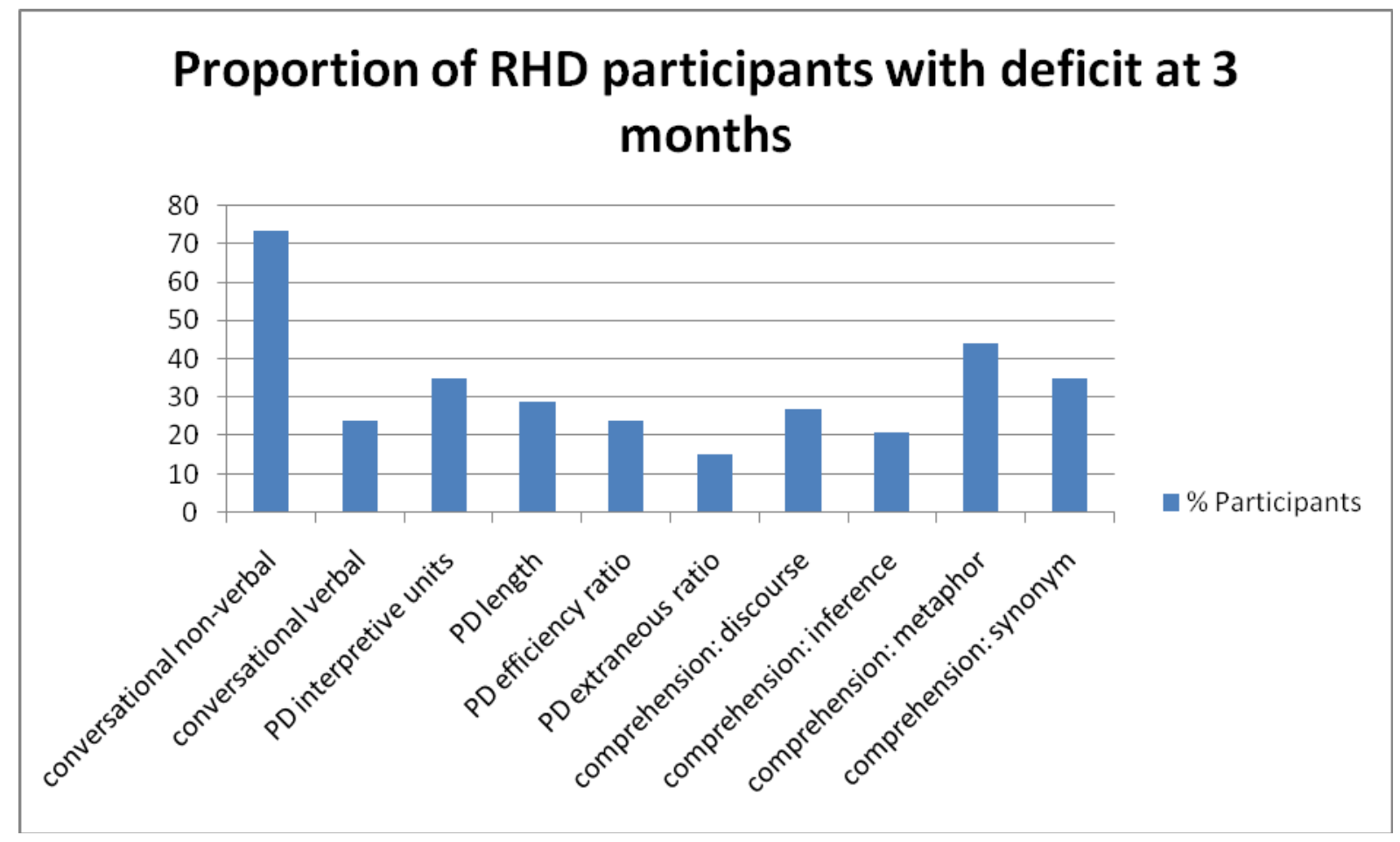

Conversational non-verbal: intonation, facial expression and eye contact measures from Rating Scale of Pragmatic Communication Skills (Burns, Halper \&Mogil, 1985);

Conversational verbal: initiation, turntaking, verbosity, topic maintenance and referencing measures from Rating Scale of Pragmatic Communication Skills (Burns, Halper \& Mogil, 1985);

PD: Picture description: cookie theft picture from Boston Diagnostic Aphasia Examination (Goodglass \& Kaplan, 1983): interpretive unit score, length $=$ word count, efficiency $=$ word count relative to content units, extraneous ratio $=$ additional unnecessary information relative to word count;

Comprehension discourse: Discourse Comprehension Test (Brookshire \& Nicholas, 1993);

Comprehension inference: Right Hemisphere Language Battery Comprehensio

Inferred Meaning Test (Bryan, 1994);

Comprehension metaphor: Right Hemisphere Language Battery Metaphor Pictur Test (Bryan, 1994);

Comprehension synonym: Psycholinguistic Assessments of Language Processing in Aphasia Auditory Synonym Judgements Test (Kay, Lesser \& Coltheart, 1992) 